\title{
Uncomplicated Pregnancy and Birth of a Healthy Mature Newborn with a Giant Tumor of Both Frontal Lobes of the Brain with Dislocation: Medical History
}

\author{
Saule Issenova ${ }^{1 \star}$, Gani Bodykov ${ }^{1,2}$, Balzira Bishekova ${ }^{1}$, Bakytkhan Kabul ${ }^{1}$, Aizhan Saparaliyeva ${ }^{1}$, Aigul Kazybayeva ${ }^{1}$ \\ 1 "National Medical University" NJSC named after S.D. Asfendiyarov, Almaty, KAZAKHSTAN \\ ${ }^{2}$ Center of Perinatology and Pediatric Cardiac Surgery, Almaty, KAZAKHSTAN \\ *Corresponding Author: isenova.s@kaznmu.kz
}

Citation: Issenova S, Bodykov G, Bishekova B, Kabul B, Saparaliyeva A, Kazybayeva A. Uncomplicated Pregnancy and Birth of a Healthy Mature Newborn with a Giant Tumor of Both Frontal Lobes of the Brain with Dislocation: Medical History. Electron J Gen Med. 2021;18(6):em336. https://doi.org/10.29333/ejgm/11337

\section{ARTICLE INFO}

Received: 29 May 2021

Accepted: 20 Sep. 2021

\section{ABSTRACT}

Background: The clinical aspect of cerebral tumor is a headache, a sign of a growing tumor is nausea, turning into vomiting, frequent dizziness, weakness, drowsiness, mental disorders such as reduced concentration, lack of appetite, a slight increase in body temperature, irritability, aggression. Pregnant women often do not attach importance to these symptoms, since they are often signs of ordinary toxicosis. We report a case of uncomplicated pregnancy, the birth of a healthy mature baby in a patient with a giant tumor of both frontal lobes of the brain and a case of maternal mortality in the postoperative period.

Aim: Aim of the article was to present a rare clinical situation - a case of maternal mortality in a 21-year-old Asian patient with uncomplicated pregnancy which had a planned cesarean section operation due to the presence of a scar on the uterus under epidural anesthesia. On the 2nd postoperative day according to MRT newly diagnosed inoperable giant tumor of both frontal lobes of the brain with a dislocation, which caused the death of the puerpera.

Results: probability of the physiological course of pregnancy and the birth of a healthy newborn in the presence of a giant meningioma of the brain (anterior frontal lobe).

Case presentation: A case of maternal mortality in a 21-year-old Asian patient with uncomplicated pregnancy which had a planned cesarean section operation due to the presence of a scar on the uterus under epidural anesthesia. On the 2 nd day of the postoperative period, an inoperable giant tumor of both frontal lobes of the brain with dislocation was diagnosed according to MRT data.

Conclusion: The combination of pregnancy and cerebral tumor is rare, in this case, it is reported about the asymptomatic course of an inoperable cerebral tumor of gigantic size. The diagnosis was confirmed by noninvasive diagnostic methods (MRT). Knowledge of this rare brain disease in pregnant women is important in the differential diagnosis of patients with mental and neurological conditions in the postpartum period.

Keywords: tumor, meningioma, pregnancy

\section{INTRODUCTION}

One of the most topical issues of obstetric sciences and practice is to study the peculiarities of pregnancy, childbirth, postpartum period, the state of the fetus and newborns in patients with severe extragenital diseases, which are one of the main causes of maternal mortality in the world and Kazakhstan. The combination of pregnancy and cerebral tumor is rare. In Kazakhstan, according to S. Akshulakov, according to statistics, maternal mortality in patients with cerebral tumors reaches $24 \%$. Such combinations as cerebral tumor and pregnancy occur 1 case per 13000-17000 [1]. There is evidence that in $75 \%$ of cases, tumors develop at a reproductive age and can make themselves felt during pregnancy for the first time, since during pregnancy there is a hormonal reorganization of the body, where the placenta is a powerful hormonal organ and provokes tumor growth [1]. The article presents a clinical case of detection of a giant tumor of both frontal lobes of the brain in a patient with a scar on the uterus, with the manifestation of the clinic in the postoperative period, remaining clinically "invisible" during the entire gestational period.

\section{CASE PRESENTATION}

A clinical case of a 21-year-old Asian patient with uncomplicated pregnancy who was admitted to the maternity hospital for a planned cesarean section with a diagnosis of 38 weeks and 3 days of pregnancy. Scar on the uterus after one cesarean section. The first pregnancy in 2016 ended with a premature operative birth due to severe preeclampsia, the 
child was 700.0, died on the 3rd day. This pregnancy is the second, registered for pregnancy at a period of 9 weeks. At 14 weeks of pregnancy, anemia benign was diagnosed (hemoglobin $104 \mathrm{~g} / \mathrm{l}$ ), received GinoTardiferon 1 tablet per day. In the first half of pregnancy, the therapist assigned antibiotic therapy for acute respiratory illness and acute pharyngitis. Thus, the first and second half of pregnancy proceeded without complications.

From the anamnesis it was found out that the patient's mother in February 2017 underwent surgery for a cerebral tumor in the occipital region.

On examination, the general condition is satisfactory. No complaints were filed. No headache, dizziness. Vision is clear. In consciousness, adequately answers questions, oriented in space, in time. The skin and visible mucous membranes are clean, normal in color. In the lungs, vesicular breathing, no wheezing. Respiration rate is 16 per 1 minute. Heart sounds are clear, rhythmic. Pulse is 74 beats per 1 minute, rhythmic. Blood pressure is $90 / 60 \mathrm{MmHg}$. The tongue is clean, moist. The abdomen is enlarged due to the pregnant uterus, soft, painless. The liver and spleen are not palpable. The symptom of "striking" is negative on both sides. The uterus is ovoid, not excitable, there is no local tenderness on palpation, and the area of the alleged scar on the uterus is painless. The position of the fetus is longitudinal; there is a head above the entrance to the small pelvis. The fetal heartbeat is clear, rhythmic, 140 beats in 1 minute. No peripheral edema. Urination is free, painless. The defecation is regular. There is no pathological discharge from the genital tract.

In a planned manner, a laparotomy was performed according to Pfannenstiel with excision of the skin scar. Caesarean section in cross section in the lower segment of the uterus. On prosection of the abdominal cavity, an adhesion process was noted, the bladder was soldered to the lower segment of the uterus. Adhesions are dissected by blunt and sharp way. At 6 minutes, a live mature female fetus weighing 3472.0, $53 \mathrm{~cm}$ tall with an Apgar score of 7 points, after 5 minutes 8 points was extracted under the axillae. By pulling the umbilical cord, removed the afterbirth of 23.0 * 25.0 * $3.0 \mathrm{~cm}$. Total blood loss - 600.0. Anesthesia - epidural anesthesia.

Attention was drawn to the increased smile of the patient when entering planned delivery, which was regarded as a positive emotional mood for the upcoming cesarean section operation and pregnancy after the loss of the fetus in the early neonatal period.

On the second day of the postoperative period, the puerpera had a clinic of psychomotor agitation: excessive maternal anxiety, excessive care, anxiety and fear for the state of health and the life of a calmly sleeping child, refusal to breastfeed. In this connection, a consultation with a psychiatrist is scheduled.

Consultation of a psychiatrist: psychological status oriented, contact, mood - low with a slight motor and ideational inhibition, but at the same time on the face of a constant formal smile. The diagnosis: Postpartum psychosis. Recommended: sonapax $10 \mathrm{mg}^{\star} 2$ times a day, amitriptyline 25 $\mathrm{mg}{ }^{\star} 3$ times a day, brusepam 2.0 intramuscularly $(10 \mathrm{mg}) \# 1$. At the time of treatment to exclude breastfeeding. Spent the night quietly, slept. No complaints were filed.

Ultrasonography of the pelvic organs. Conclusion: Postoperative period is 3 days. Ultrasonography of the abdominal cavity - there is no free fluid.
On the third day, against the background of complete rest, there is a sharp deterioration in the condition, the clinical picture of acute cerebral circulatory disorders in connection with which was transferred to the ARITD.

The condition is considered as bad. Consciousness - sopor with the transition to a coma. On the scale of Glasgow - 8-9 points. The pupils are moderately dilated. $\mathrm{D}=\mathrm{S}$, the photo reaction is sluggish. For the purpose of neurovegetative protection, she was transferred to ALV. The right jugular vein was punctured and catheterized.

Consulted by a neurologist: Toxic encephalopathy. Brain edema. Poisoning with amitriptyline? CVA? Recommended: MRT of the brain, consultation of a toxicologist, oculist, kontrykal 1000,0 intravenous, drip, mannitol $200,0-15 \%$ on a saline solution of sodium chloride, intravenous, drip.

Consulted by an ophthalmologist: Fundus of eye without pathology.

Consulted by a toxicologist: There is no data for amitriptyline poisoning. The urine test for amitriptyline is negative.

Repeated consultation of a psychiatrist: In connection with the patient's condition, the prescribed treatment should be canceled.

Analysis of cerebrospinal fluid: Conclusion: without pathology.

Ultrasonography of the abdominal cavity organs: Conclusion: there is no free fluid in the abdominal cavity.

MRT of the brain: Conclusion: MRT-scan of the formation of the left frontal lobe, with a high probability of intracerebral hematoma (acute stage), not excluding tumor formation with hemorrhage, signs of transtentorial herniation (Figure 1).

Consulted by a neurosurgeon: Meningioma of the anterior third of the falx with bilateral growth, with hemorrhage in the tumor. Axial misalignment. An inoperable tumor.

Consulted by a stroke specialist: Volume formation of the left frontal lobe, complicated by hemorrhage.

Despite intensive therapy, administration of vasotonic and cardiotonic drugs, cardiac arrest occurred against the background of the therapy.

Postmortem diagnosis: Giant tumor of both frontal lobes of the brain with dislocation, complicated by hemorrhage in the tumor. Coma III, respiratory depression, depression of the cardiovascular center.

Cause of the death: Edema, swelling of the brain. Dyshemic disorders of brain stem. Acute respiratory and cardiovascular insufficiency.

Dislocation syndrome. Herniation of the brain. Edema, swelling of the brain. Dyshemic disorders of brain stem.

Pathomorphological conclusion: Giant cerebral tumor with localization in the left frontal-parietal region (meningothelial meningioma with foci of anaplastic structure with sprouting of the Dura mater, secondary changes in the form of massive hemorrhages, necroses, foci of sliming) (Figure 2).

\section{DISCUSSION}

According to literature sources [2,3,4], the development of cerebral tumors in pregnant women is associated with the 

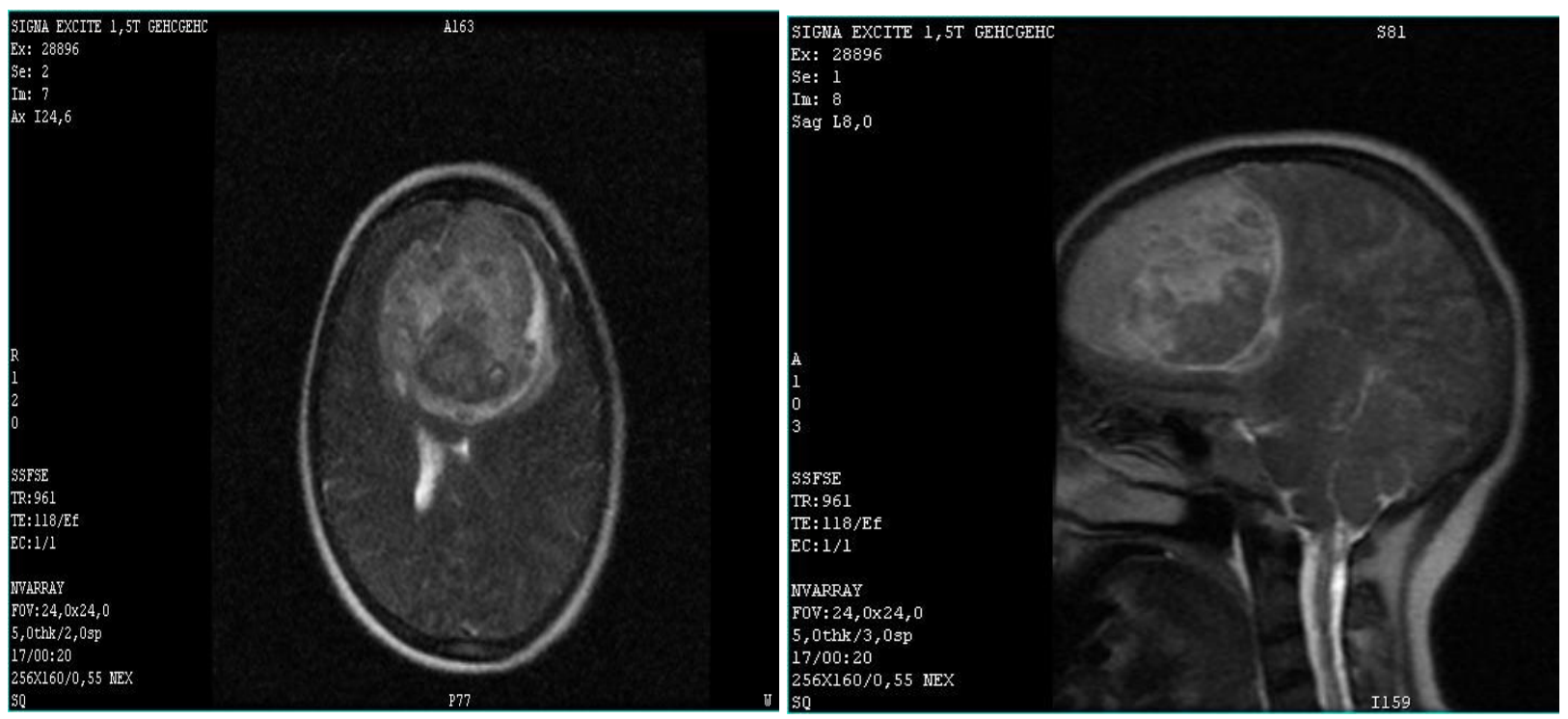

Figure 1. MRT-scan of the formation of the left frontal lobe, with a high probability of intracerebral hematoma

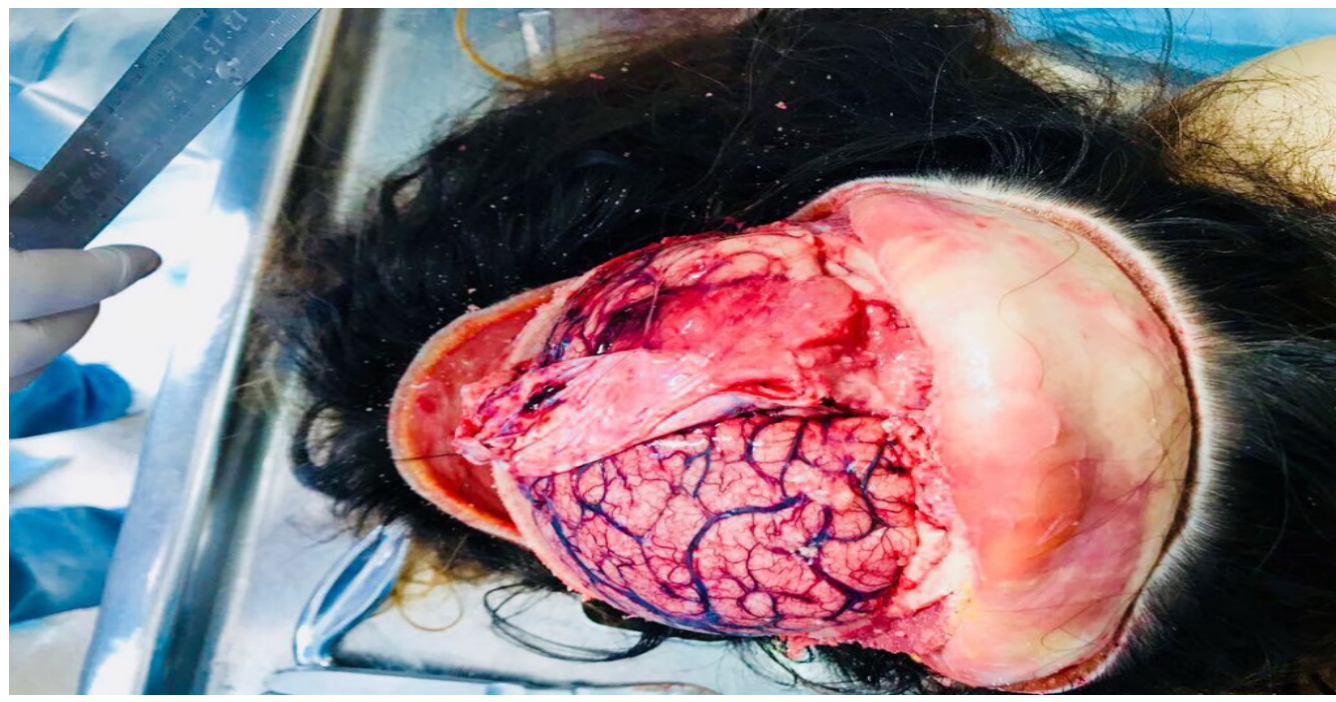

Figure 4. Pathomorphological picture of a giant cerebral tumor

following provoking factors: transferred or active cancer of other vital organs; radiation acting on a healthy body; the constant presence of artificial additives in the diet; the impact of toxins on the body; regular and prolonged exposure to direct sunlight; exposure to electromagnetic waves from technology and gadgets; hereditary predisposition. The possibility of developing brain cancer due to an inherited factor is still an open question. To date, experts cannot confirm the influence of heredity on the appearance of cancer, but they do not refute this fact.

It is known that the clinical picture of the disease depends on the location and size of the tumor when the tumor in the frontal lobe may exhibit the following clinical symptoms: mental deficiency, silliness (behavior, characterized by silly jokes, babbling), speech disorders (slurred speech of patient, like "porridge in the mouth", pulling lips into a tube (as in sucking) spontaneously or when touch something to lips, unsteadiness of gait - often the patient during walking has a tendency to fall back. Changes in a person's behavior and character are especially characteristic, but they are usually associated with stress, some changes in life. Therefore, they are not given due importance. And only with the appearance of sudden changes in the psyche or addition of other symptoms (visual disorder, epileptic seizures), the patient seeks medical help [5].

This case report provides a few learning points:

- asymptomatic course of giant meningioma of the brain (anterior parts of the frontal lobe);

- increased smile of the patient when entering planned delivery, which was mistakenly interpreted as a positive emotional mood for the upcoming cesarean section operation and pregnancy after previous fetal loss in the early neonatal period;

- presence of a giant tumor and epidural anesthesia during cesarean section did not lead to anaesthetic complications and side effects intraoperatively and in the early postoperative period;

- epidural anesthesia may have been the starting point in the manifestation of the clinical picture of a cerebral tumor with dislocation and herniation;

- the rapid change of symptoms of arousal and inhibition was a symptom of brain herniation, edema, swelling of 
the brain, as well as dyshemic disorders of the brain stem;

- excessive maternal anxiety, excessive care, anxiety and fear for the health and life of a calmly sleeping child, refusal to breastfeed were the first symptoms of dislocation of a cerebral tumor and were mistakenly accepted as a manifestation of postpartum psychosis;

- possibility of a physiological course of pregnancy in giant meningioma of the anterior frontal lobe of the brain;

- probability of giving birth to a healthy mature newborn in a patient with a giant meningioma of the brain;

- presence of a cerebral tumor in the mother does not exclude the possibility of a hereditary family predisposition to the development of a cerebral tumor;

- it is possible that the cerebral tumor was also present during the previous pregnancy and was a manifestation and/or cause of preeclampsia.

\section{CONCLUSION}

We present a rare clinical case of maternal mortality in a 21year-old Asian patient with uncomplicated pregnancy which had a planned caesarean section due to the presence of a scar on the uterus under epidural anesthesia. On the 2nd postoperative day according to MRT newly diagnosed inoperable giant tumor of both frontal lobes of the brain with a dislocation, which caused the death of the puerpera. The presented case testifies to the probability of the physiological course of pregnancy and the birth of a healthy mature newborn in a patient with a giant meningioma of the brain. The presence of a cerebral tumor in the mother does not exclude the possibility of a hereditary family predisposition to the development of a cerebral tumor.

Author contributions: All authors have sufficiently contributed to the study, and agreed with the results and conclusions.

Funding: No funding source is reported for this study.

Declaration of interest: No conflict of interest is declared by authors.

\section{REFERENCES}

1. Akshulakov SA. Surgeries to remove tumors in pregnant women are being successfully performed in Kazakhstan. 2014. Available at: https://www.zakon.kz/amp/4633815-vkazakhstane-uspeshno-prokhodjat.html

2. Klein A. (Ed.). Pregnancy and neurologic illness. Neurol. Clin. 2012;30:781-962. https://doi.org/10.1016/ j.ncl.2012.06.005 PMid:22840799

3. Nossek E, Ekstein M, Rimon E, et al. Neurosurgery and pregnancy. ActaNeurochir. 2011;153:1727-35. https://doi.org/10.1007/s00701-011-1061-y PMid:21660423

4. Arnardóttir S, Geirsson R. High Risk Pregnancy: Management Options edited by David James, Philip Steer, Carl P. Weiner, Berhard Gonik, Caroline A Crowther and Stephen C. Robson. Acta Obstetricia et Gynecologica Scandinavica. 2011;90(12):1456. https://doi.org/10.1111/ j.1600-0412.2011.01281.x

5. Powell M. Pituitary tumors and pregnancy. ActaNeurochir. 2011;153:1737-8. https://doi.org/10.1007/s00701-0111062-x PMid:21660422 